\title{
Uso dell'Analisi Qualitativa Comparata nello studio del paziente fragile.
}

\author{
Alessandro Addorisio ${ }^{1}$ \\ ${ }^{1}$ Affiliation not available
}

November 11, 2021

\begin{abstract}
La complessità del mondo della cura ha fra le sue espressioni più indagate quella del paziente fragile, nonostante la sua definizione sia ancora dibattuta. Questo studio è il tentativo di utilizzare uno strumento messo a punto in ambito sociologico, l'Analisi Qualitativa Comparata, per ridurne la complessità descrittiva. La modalità olistica di questo approccio consente, anche nel campo della cura, di cogliere l'aspetto multi-dimensionale del paziente fragile, risultando utile alla sua analisi.
\end{abstract}

Parole chiave : fragilità - AQC - tavola di verità - algebra booleana - olismo

The complexity of the world of care has among its most investigated expressions that of the frail patient, although its definition is still debated. This paper describes the attempt to use a tool developed in the sociological field, Comparative Qualitative Analysis, to reduce its descriptive complexity. The holistic modality of this approach allows, also in the field of care, to grasp the multi-dimensional aspect of the frail patient, resulting useful for his analysis.

Key words : fragility - AQC - truth table - Boolean algebra - holism

Da molti anni, la comunità medica, spinta della necessità di guardare il malato non solo come portatore di patologia ma anche di un patrimonio di motivazioni, esperienze, cultura, credenze, connessioni sociali ed ambientali, ha iniziato a riconsiderare il modo stesso di definire la malattia, le sue conseguenze, l'opportunità e perfino l'eticità di alcune pratiche di cura, soprattutto quando la malattia si inserisce in un quadro non complicato ma complesso, ossia quando la malattia stessa modifica, in modo spesso imprevedibile, una serie di fattori che non sono solo di natura biochimica o fisica ma anche psicologica, sociale, economica ed etica, determinando uno squilibrio al quale non è sufficiente rispondere con la sola terapia medica.

Sono così diventate sempre più frequenti le connessioni e gli intrecci fra discipline e materie diverse, praticate da professionisti e studiosi che dal proprio punto di vista approcciano la complessità dell'individuo malato.

Questo contributo va in questa direzione, nella convinzione che la complessità del mondo contemporaneo, compresa quella medica e sanitaria, non possa essere affrontata da prospettive singole ma dalla cooperazione fra gli studiosi di discipline scientifiche diverse.

Lo scopo di questo studio è stato quello di verificare se uno strumento di ricerca, denominato Analisi Qualitativa Comparata (AQC), utilizzato nelle scienze sociali, potesse essere utile per analizzare un fenomeno che si presenta nel campo della cura medica e che è stato definito "paziente fragile" (PF).

Il paziente fragile è oggi fra le espressioni più studiate della complessità del mondo della cura perché ne rappresenta più di altre la difficoltà di descrizione e di definizione nonché la natura multi-dimensionale, interconnessa e non di rado contraddittoria. 
La definizione di paziente fragile è tuttora un processo in evoluzione le cui tappe non riguardano le finalità di questo articolo però, sebbene manchi una versione definitiva e condivisa fra i professionisti della salute al momento, ci sono alcuni elementi che la caratterizzano e che possiamo così sintetizzare: la fragilità è una condizione che coinvolge prevalentemente soggetti anziani, affetti da patologie, disabili, con difficoltà mnemoniche e di .dizione può essere complicata da fattori socio-economici come solitudine, difficoltà relazionali o povertà.

Soprattutto in ambito geriatrico si sono fatti sforzi per estendere il concetto di fragilità a dimensioni che non siano solo quelle fisiche e cliniche2, proponendo l'utilizzo di strumenti di valutazione multidimensionale per soggetti anziani, pur riconoscendone la difficoltà di utilizzo in ambito clinico33Pilotto A., Ferrucci L., Verso una definizione clinica della fragilità:utilità dell'approccio multidimensionale, G.Gerontol. 2011, 59, pp.125-29.

Il concetto di paziente fragile, come tutti i concetti, dipende da quali casi si hanno in mente quando lo si definisce, per cui occorre porre molta attenzione ad inserire oppure omettere un criterio fra quelli che contribuiscono alla sua generalizzazione.

Questa accortezza non sembri una semplice questione di procedura teorica perché le implicazioni possono essere molto concrete se, ad esempio, le immaginiamo nella sfera delle politiche socio-sanitarie. Da qui la necessità di approfondire la conoscenza di questo fenomeno da prospettive diverse.

L'dea di partenza è stata quella di valutare la fragilità di un paziente non come unica condizione, quale viene considerata dal punto di vista medico44De Toni A., Giacomelli F., Ivis S., Il mondo invisibile dei pazienti fragili, Utet Università, 2010, ma come una configurazione di condizioni, in altre parole che la fragilità sia esaminata come un tutto, le cui cause sono collegate fra loro e possono essere analizzate solo nel contesto del caso e non separatamente come spesso avviene.

In genere, in ambito medico, la fragilità viene catalogata fra i fenomeni complessi 55(ibidem), ossia quelli nei quali le variabili sono molte e le relazioni fra esse non sono di tipo lineare. In generale però, aldilà dell'azione classificatoria, l'assunzione di una tecnica di indagine basata sulle singole variabili non appare idonea a ridurre la complessità di analisi di questo fenomeno.

Come appare evidente nelle definizioni su riportate, spesso vengono prese in esame le variabili che contribuiscono, con grado diverso, a determinare la condizione di fragilità. In altre parole, la fragilità è considerata l'output delle variabili anzianità, multi-patologie croniche, disabilità, solitudine, ecc.

Nelle ricerche che si basano sulle tecniche statistiche di indagine multivariata si cercano proprio le cause multiple congiunturali, o meglio, si cerca di stimare il contributo di ciascuna causa. Le singole cause possono, indipendentemente l'una dall'altra, incrementare o ridurre la probabilità che un certo risultato si verifichi oppure il suo livello di intensità o la sua consistenza.

Ipotizziamo che da un'indagine di questo tipo emerga che un problema di salute, ad esempio il diabete, aumenti del 10\% la possibilità del paziente di diventare fragile e che un altro problema, la solitudine, aumenti tale probabilità del 20\%. La conclusione che ne può coerentemente derivare è che il diabete sommato alla solitudine comporti per il paziente un aumento del $30 \%$ della probabilità di diventare fragile. Ma stimare il contributo di ogni singola causa indipendentemente dalle altre non raggiunge l'obiettivo di trovare la configurazione di condizioni che determinano la fragilità.

Per le finalità della medicina, stimare in termini probabilistici la possibilità che un determinato evento, ad esempio una malattia, accada è di fondamentale importanza e questo tipo di approccio ha contribuito a produrre nel corso del secolo scorso i grandi successi raggiunti, sia nella prevenzione che nella terapia, però questo metodo non è altrettanto efficace nell'analisi di situazioni complesse nelle quali le variabili interferiscono l'una con l'altra e spesso non appartengono nemmeno allo stesso dominio, come nell'esempio sopra citato.

La solitudine, la scarsità di risorse economiche o le difficoltà relazionali non sono pertinenza della scienza 
medica ma delle scienze sociali, politiche o psicologiche.

E' per questo che si è pensato di utilizzare, in una situazione complessa come quella del cosiddetto paziente fragile, un approccio che è stato sviluppato nel campo della sociologia. Questo vuol dire anche aggiungere una dimensione sociale ad un fenomeno considerato prevalentemente dal punto di vista medico.

Definire fenomeni, situazioni o condizioni complesse comporta inevitabilmente il tener conto di alcuni aspetti e di trascurarne altri per non affondare in un mare di dettagli ma, fra la perfezione di un modello lineare nel quale A è causa di B e l'indescrivibilità del caos esistono molti stati intermedi che vale la pena indagare.

Provare ad aggiungere ulteriori elementi che contribuiscono a definire un fenomeno o una situazione, non vuol dire necessariamente aumentarne la complessità, anzi, si possono adoperare metodi che servono a utilizzare ciò che è già noto per scoprire nuove dimensioni attraverso l'uso della logica.

Uno di questi metodi è stato descritto da Lazarfeld e Barton, i quali così suggeriscono di affrontare tali situazioni:

" [..] l'analisi delle osservazioni qualitative deve affrontare una massa di fatti particolari di tal numero e varietà che non sembra pratico trattarli separatamente [..]. In tale situazione il ricercatore elaborerà un concetto descrittivo ad un livello superiore che cercherà di raggruppare e riassumere una grande abbondanza di osservazioni particolari in una singola formula ." 66Lazarsfeld, P.F., Metodologia e ricerca sociologica ,354-356, Il Mulino, 1967.

Un tale concetto descrittivo è quello di paziente fragile che esprime le caratteristiche del paziente in una formula sintetica, in una tipologia empirica formata a partire dalle combinazioni dei valori delle variabili.

Costruire una tipologia vuol dire dare un nome unico ad una varietà di situazioni o cose che stanno insieme in modo frequente.77Becker H.S., Tricks of the Trade, University of Chicago Press, 1998, ed it. I trucchi del mestiere, Il Mulino, 2007 I metodi utilizzabili per ottenere queste tipologie sono concepiti per gestire e utilizzare la varietà delle situazioni rilevabili empiricamente.

Si è già accennato all'inefficacia di alcune tecniche statistiche nell'analisi di fenomeni complessi, nei quali più che il contributo di ciascuna variabile al risultato che interessa, è importante individuare le configurazione dei fenomeni che, variamente combinati, producono il risultato stesso. A tal scopo Charles Ragin ha sviluppato il metodo dell'Analisi Qualitativa Comparata88Ragin C.C., The Comparative Method: Moving beyond Qualitative and Quantitative Strategies, University of California Press, 1987. detta anche analisi booleana perché basata sull'algebra logica elaborata dal matematico inglese George Boole.

Uno strumento fondamentale di questo metodo logico va sotto il nome di tavola di verità e serve a ridurre la complessità della gestione di molte configurazioni di situazioni, ossia di variabili, con il grande vantaggio di evitare il rischio di ometterne per errore qualcuna, in quanto la logica soggiacente al metodo garantisce che, con quelle variabili, non ci sono e non possono esserci altri tipi di configurazione oltre a quelli individuati. 99Becker H.S. (op.cit.)

Un metodo probabilmente più conosciuto è quello che prevede la costruzione di tabelle a doppia entrata nelle quali le variabili si incrociano fra loro per determinare le tipologie possibili. Per fare un esempio (tab.1), scegliamo due variabili, esposizione ad un fattore di rischio e sviluppo di una malattia, e le facciamo incrociare dando luogo a quattro tipologie: malato esposto, sano esposto, malato non esposto e sano non esposto.

\begin{tabular}{lll}
\hline ESPOSIZIONE & MALATTIA SI & MALATTIA NO \\
\hline SI & Malato esposto & Sano esposto \\
NO & Malato non esposto & Sano non esposto \\
\hline
\end{tabular}

Tab.1

Questa stessa tabella può essere presentata in termini di tavola di verità (tab.2) nella quale le variabili sono 
indicate in colonna e le quattro tipologie sono caratterizzate dalla presenza o dall'assenza di una delle due variabili o di entrambe.

Tavola di verità ( $1=$ presenza, $0=$ assenza $)$

\begin{tabular}{ll}
\hline ESPOSIZIONE & MALATTIA \\
\hline+ & + \\
+ & - \\
- & + \\
- & - \\
\hline
\end{tabular}

Tab.2

In questa tavola di verità, la prima riga rappresenta il malato esposto, la seconda il sano esposto, la terza il malato non esposto e la quarta riga il sano non esposto.

Da un punto di vista logico non ci sono altre tipologie possibili e se anche nella ricerca empirica una di queste tipologie non fosse ritrovata, cioè non venissero trovati casi, ciò non vorrebbe dire che essa non esiste.

Quando le variabili sono più di due, la tavola di verità non solo riesce a rappresentare graficamente le combinazioni in maniera più comprensibile ma soprattutto garantisce che quelle previste siano le sole possibili.

L'utilizzo della tavola di verità è legato a questa considerazione: quando esaminiamo un oggetto possiamo attribuirgli alcune caratteristiche, riconosciute come rilevanti, ad esempio peso o durezza e da quel momento tutti gli oggetti di quel tipo possiederanno un valore per quella caratteristica, compreso il valore 0. La distinzione da comprendere è fra l'esistenza di una proprietà e il valore 0 di quella proprietà. Se il valore è 0 non vuol dire che quella proprietà rilevante non esista più perché l'oggetto continua ad averla pur con un valore nullo. Nel caso di un paziente fragile, noi possiamo considerare come sue proprietà rilevanti un parametro bio-chimico, un certo deficit oppure una caratteristica sociale come la solitudine. Non conosciamo sempre tutte le caratteristiche che possiamo attribuire al paziente fragile, ma quando ne troviamo uno che possiede una caratteristica particolare che lo distingue dagli altri allora sappiamo che tale caratteristica esiste e possiamo vedere che anche gli altri pazienti fragili la possiedono, con valori diversi, fino all'estremo del valore 0 , ossia della sua assenza. 11Becker H.S. (op.cit.)

Proviamo con un esempio a rendere meglio il concetto: se si decide, in genere lo fa la comunità scientifica o professionale, che la solitudine è una caratteristica del paziente fragile allora tutti i pazienti fragili avranno questa caratteristica, anche coloro per i quali è assente. Per questo motivo è importante l'uso della tavola di verità che verifica non solo la presenza ma anche l'assenza di tutte le caratteristiche. Nell'analisi booleana infatti l'assenza di una caratteristica, di un fattore causale, di una condizione, riveste la stessa importanza della sua presenza, a causa del carattere olistico di questo metodo che prende in considerazione l'insieme, il tutto.

Il tentativo di questo studio è verificare se l'Analisi Qualitativa Comparata può essere uno strumento adeguato a gestire la complessità che si presenta quando si vuole far emergere la varietà possibile contenuta nella tipologia del PF, vista dalla prospettiva del Medico di Medicina generale (MMG).

La scelta di tale prospettiva è dovuta alla convinzione che il MMG sia il professionista della salute più indicato per cogliere la situazione complessa di questa tipologia di paziente, in quanto a conoscenza non solo del quadro clinico del paziente, quindi delle variabili biologiche, ma anche della sua situazione familiare, ambientale, sociale e psicologica, cioè delle variabili psico-sociali.

Per costruire una tavola di verità utile allo scopo, per prima cosa si è cercato di individuare le variabili che danno come risultato la condizione di paziente fragile. Le variabili sono state scelte basandosi su colloqui con MMG e su dati pubblicati.22De Toni et al. (op.cit) 
Le variabili di tipo nominale utilizzate sono state trattate come dicotomie perciò avevano due possibilità di stato, espresso come presenza o assenza.

La matrice dei dati è stata costruita in forma di tabella le cui righe rappresentavano i casi empirici di PF raccolti e le colonne il valore assunto dalle variabili, codificato in 1 per la presenza e 0 per l'assenza. In questo modo, ogni singola cella della tabella corrispondeva alla presenza o assenza della variabile indicata in colonna, incrociata con il caso di PF della riga.

Le variabili presenti in letteratura oppure citate dai MMG durante i colloqui, erano in numero superiore a quelle utilizzabili nello studio, per cui si è proceduto ad un lavoro preliminare di semplificazione e di accorpamento.

Il motivo di questa semplificazione deriva dalla natura matematica della tavola di verità poiché per ogni variabile aggiunta le possibili combinazioni fra le stesse aumentano con un tasso di 2.

Ad esempio, se le variabili esaminate sono due le possibili combinazioni fra esse sono: $2 \times 2=4$, mentre se le variabili sono tre le combinazioni diventano $2 \times 2 \times 2=8$, se quattro le combinazioni sono $2 \times 2 \times 2 \times$ $2=16$ e così via. Per evitare di trovarsi a gestire un numero troppo elevato di combinazioni si è deciso di considerare fino a tre variabili.

E' possibile naturalmente utilizzare un numero maggiore di variabili ma la complessità di calcolo aumenta in maniera esponenziale e per gli scopi di questo studio si è ritenuto che tre variabili fossero sufficienti a verificare se il metodo utilizzato, l'analisi booleana, sia utile ed adeguato ad affrontare la complessità presentata dal paziente fragile.

Si è provveduto quindi ad inviare ai MMG partecipanti una scheda di rilevazione che comprendeva sei variabili causali per poter raccogliere, almeno inizialmente, una gamma più ampia di possibilità. Successivamente le sei variabili sono state accoppiate, per raggiungere il numero prefissato di tre.

Approntata la scheda (fig.1), è stata inviata a dieci MMG che si erano resi disponibili, con la richiesta di individuare fra i propri assistiti alcuni casi di PF e quindi indicare per ciascuno di essi, con una risposta affermativa o negativa, la presenza oppure l'assenza delle variabili.

\begin{tabular}{llllll}
\hline PAZIENTE & MULTIPATOLOGIA & MULTITERAPIA & AUTONOMIA & CAPACITA' ECONOMICA & CONVIVENTI \\
\hline sigla & SI $/$ NO & SI/NO & SI/NO & SI/NO & SI /NO \\
DP & SI $(1)$ & SI $(1)$ & NO $(0)$ & SI $(1)$ & SI $(1)$ \\
BG & SI $(1)$ & SI $(1)$ & SI $(1)$ & SI $(1)$ & NO $(0)$ \\
\hline
\end{tabular}

\section{Scheda di rilevazione}

fig.1

La matrice ottenuta conteneva 39 casi di PF, non equamente ripartiti fra i cinque MMG che hanno risposto poiché alcuni di essi ne hanno individuati più degli altri, ma la numerosità dei casi, differentemente dalle analisi di tipo statistico quantitativo, non ha qui importanza se non quella di assicurare una maggiore varietà di possibili configurazioni di variabili. Ricordiamo che tale varietà ha un limite massimo, matematicamente determinato dal numero delle variabili considerato, che può essere o meno raggiunto a seconda del numero di casi di PF indicati dai rispondenti.

La prima variabile considerata è stata la polipatologia, ossia la presenza, nel quadro clinico del paziente fragile, di più malattie.

La seconda variabile riguardava la politerapia eventualmente seguita dal PF, considerando anche quella fisiatrica.

Queste prime due sono state accorpate in un'unica variabile (B ) perché entrambe, aldilà della specificità 
in un quadro clinico, rappresentano la complessità della patologia dal punto di vista medico che non può mancare in un'analisi sul paziente fragile.

Il valore 1 (presenza) assegnato a questa variabile corrisponde alla presenza di almeno una delle variabili accorpate, polipatologia o politerapia, poiché sono state considerate entrambe condizioni sufficienti anche se non necessarie per indicare la complessità clinica del paziente fragile.

La terza variabile della matrice è stata la capacità di autogestione del paziente, ossia l'autonomia nella vita quotidiana. Una eventuale disabilità del $\mathrm{PF}$, di qualsiasi natura, viene ad essere così ricompresa in questa variabile. Qualcuno potrebbe obiettare che questa variabile sarebbe meglio rappresentata da valori che esprimano il grado di autonomia su una scala numerica ma ai fini dello studio non è necessario stabilire quanto sia l'autonomia poiché, dato che il MMG lo ha indicato come un caso di PF, ciò che interessa è se, per quel caso specifico, l'autonomia era o meno presente. Ribadiamo qui che lo scopo dello studio non è indagare quanto ciascuna variabile contribuisca al risultato finale, cioè la fragilità, ma quali configurazioni di variabili risultano presenti quando si ha quel risultato.

La quarta variabile è stata dedicata ad indagare la capacità economica del PF di acquistare beni o servizi di cura per sé.

La terza e la quarta variabile sono state accorpate poi in un'unica variabile (A ), il cui valore 1 (presenza) indica una piena autonomia del paziente, sia gestionale che economica, mentre il valore 0 (assenza) indica la mancanza di almeno una delle due condizioni.

La quinta variabile è stata individuata nella presenza o meno di conviventi con il PF, compresa quella di eventuali cosiddetti "badanti", perché, a prescindere dal tipo di vincolo che unisce il convivente al paziente, che sia affettivo o economico, si vuole esplorare la condizione di solitudine.

Infine l'ultima variabile riguardava la presenza di una rete sociale attiva, costituita indifferentemente da congiunti, amici o vicini che regolarmente supportassero il PF. Quest'ultima è stata poi considerata unitamente alla quinta, sulla convivenza, per dar luogo ad un'unica variabile (S ) che potremmo riferire alla condizione di solitudine che può essere alleviata o aggravata dalla presenza o meno di una rete sociale attiva.

Dal punto di vista dei valori da attribuire a questa variabile, 1 per la presenza e 0 per l'assenza della solitudine, essi dipendevano dall'assenza contestuale di conviventi e della rete sociale, in altri termini la condizione di solitudine è stata ritenuta presente, valore 1 , se entrambe le variabili accorpate avevano valore 0 , assenza di conviventi e di rete sociale, mentre la solitudine è stata considerata assente se entrambe o almeno una delle suddette variabili risultava presente.

Le sei variabili originarie presentate nella scheda di rilevazione sono state così ridotte a tre $: \mathrm{B}, \mathrm{A}$ ed $\mathrm{S}$.

Come già detto, per poter gestire le combinazioni possibili fra le variabili si è deciso di limitare a tre il numero delle variabili. In questo modo, il numero massimo di combinazioni è uguale a 8 , mentre se avessimo considerato il gruppo intero delle sei variabili il numero teorico delle possibili combinazioni sarebbe salito a 64, molto più complicato da gestire. Esistono procedure e anche software che consentono di affrontare combinazioni di variabili più numerose ma per gli scopi di questo studio sarà sufficiente il numero indicato.

Nelle pagine seguenti si è indicato con la lettera maiuscola la presenza della variabile (valore 1) e con la lettera minuscola la sua assenza (valore 0). Quindi, ad esempio, A indicherà la presenza dell' autonomia per quel caso specifico di PF e a ne indicherà invece l'assenza. Come detto in precedenza, nell'analisi booleana sia la presenza che l'assenza di una variabile ricoprono la stessa importanza perché è l'insieme, il tutto, che viene considerato. Alcuni casi possono avere la stessa configurazione di variabili, altri differire per alcune o tutte le variabili ma tutti condividono la variabile dipendente, ossia la condizione di paziente fragile. Ottenute le risposte, la matrice dei dati è stata reimpostata come tavola di verità ( fig.2) elencando le configurazioni delle variabili che producono il risultato PF.

Tavola di verità ( $1=$ presenza, $0=$ assenza $)$ 


\begin{tabular}{lllll}
\hline COMBINAZIONI & VARIABILE & VARIABILE & VARIABILE & VARIABILE \\
\hline & $\mathrm{B}$ & $\mathrm{A}$ & $\mathrm{S}$ & $\mathrm{PF}$ \\
1 & 1 & 1 & 1 & 1 \\
2 & 1 & 0 & 1 & 1 \\
3 & 1 & 1 & 0 & 1 \\
4 & 1 & 0 & 0 & 1 \\
5 & 0 & 1 & 1 & 0 \\
6 & 0 & 0 & 1 & 0 \\
7 & 0 & 1 & 0 & 0 \\
8 & 0 & 0 & 0 & 1 \\
\hline
\end{tabular}

Fig.2

$\mathbf{B}$ : complessità clinica $\mathbf{A}$ : autonomia $\mathbf{S}$ : solitudine

La tavola di verità può essere così espressa in termini algebrici booleani :

$\mathrm{PF}=\mathrm{BAS}+\mathrm{BaS}+\mathrm{BAs}+\mathrm{Bas}+\mathrm{bas}$

Ciascuno dei prodotti indicati nella formula rappresenta una combinazione delle variabili trovate nei casi di PF elencati dai MMG.

Occorre precisare che nell'algebra booleana la moltiplicazione e l'addizione non sono aritmetici per cui l'espressione BAs non vuol dire che il valore di B vada moltiplicato per quello di A e poi per quello di s per dare il risultato $\mathrm{PF}$, ma significa che la presenza della variabile B è combinata con la presenza della variabile A e con l'assenza della variabile $s$, formando la configurazione BAs che dà luogo a PF .

In altri termini, nell'algebra booleana il segno di moltiplicazione indica l'operatore logico AND (congiunzione) mentre il segno di addizione indica l'operatore logico OR (disgiunzione) perciò la somma di prodotti su riportata significa che le tre variabili causali sono congiunte (AND) in differenti configurazioni che danno in modo disgiunto (OR) lo stesso risultato PF.

\section{Minimizzazione delle espressioni booleane .}

Le operazioni svolte fino a questo punto potrebbero far pensare ad un aumento della complessità piuttosto che ad una sua riduzione ma ora vedremo che questo non è vero.

Innanzitutto occorre applicare una regola fondamentale dell'approccio booleano la quale afferma che se due espressioni booleane differiscono per una sola variabile ma producono lo stesso risultato allora la variabile che distingue le due espressioni può essere considerata irrilevante e rimossa per creare un'espressione combinata più semplice.11Ragin C.C., (op.cit, pag. 93)

Considerando le prime due righe della tavola di verità (fig.2), vediamo che differiscono solo per la variabile A che nella riga 2 è assente (valore 0 ) e nella riga 1 è presente (valore 1), perciò le due espressioni $\mathbf{B a S}$ e BAS , applicando la regola su citata possono essere semplificate nell'espressione BS .

In altre parole, il confronto fra le due espressioni, considerate in modo olistico, indica che la variabile A è ininfluente perché comunque il risultato è $\mathrm{PF}$.

Il processo di minimizzazione è applicato secondo una procedurabottom-up, fin quando non è possibile compiere ulteriori semplificazioni.

Procediamo con la minimizzazione dell'espressione booleana su citata:

BAS unita con BaS si riduce a BS

BAS unita con BAs si riduce a BA 
Bas unita con bas si riduce a as

L'espressione ridotta che si ottiene è la seguente:

$\mathrm{PF}=\mathrm{BS}+\mathrm{BA}+\mathrm{as}$

Questa procedura mostra come, mediante la minimizzazione booleana, sia possibile individuare un'espressione più semplice di condizioni causali. Infatti, le iniziali cinque combinazioni di tre variabili sono state ridotte a tre combinazioni di due variabili.

\section{Necessario e sufficiente}

I risultati della procedura di minimizzazione consentono anche di valutare le espressioni causali in termini di necessità e sufficienza.11(ibidem)

Affinché una causa $x$ sia considerata necessaria per il risultato $y$ occorre che se $x$ non è vera, cioè non accade, il risultato $y$ non avviene, ossia $x$ deve essere presente perché si ottenga $y$. Formalmente si esprime con $x y$ , cioè $x$ implica $y$.

Una causa $x$ è definita sufficiente se da sola può produrre un certo risultato $y$. Formalmente $y x$, ossia yimplica $x$.

Una causa è considerata necessaria e sufficiente se essa è l'unica causa di quel risultato ed è singola, cioè non ha bisogno di altre cause.

Una causa può essere sufficiente ma non necessaria se è in grado di produrre un certo risultato ma non è la sola causa con questa capacità.

Viceversa, una causa può essere necessaria ma non sufficiente se è capace di produrre un risultato assieme ad altre cause e compare in tutte le combinazioni.

Infine, una causa può non essere né necessaria né sufficiente se è presente solo in un sotto-gruppo di combinazioni di cause che producono un risultato.

Consideriamo ora l'equazione risultata dalla ricerca:

$\mathrm{PF}=\mathrm{BS}+\mathrm{BA}+\mathrm{as}$

Nessuna delle cinque condizioni causali ( $\mathrm{B}, \mathrm{S}, \mathrm{A}, \mathrm{a}, \mathrm{s})$ risulta essere né necessaria né sufficiente a produrre il risultato PF poiché appare evidente che tutti e tre i termini dell'equazione sono delle combinazioni di cause.

Questa considerazione può essere interessante perché a volte si tende a sovrastimare un fattore causale per la sua frequenza nei casi empirici riscontrati, al punto di ritenerlo necessario, mentre è solo sufficiente, oppure il contrario.

Solo a titolo esemplificativo, se dall'equazione eliminassimo l'ultimo termine as, cioè la mancanza di autonomia pur in presenza di conviventi, la formulazione diventerebbe:

$\mathrm{PF}=\mathrm{BS}+\mathrm{BA}$

nella quale B, ossia la presenza di polipatologia o politerapia o entrambe, è causa necessaria ma non sufficiente in quanto è presente in tutte le combinazioni ma mai da sola.

\section{Primi implicanti}

Un'altra fase fondamentale dell' analisi booleana è trovare i cosiddettiprimi implicanti .11(ibidem)

Si dice che un'espressione booleana ne implica un'altra se appartenere alla seconda corrisponde ad appartenere ad un sottogruppo della prima. Per esempio, BS è un primo implicante che implica BAS e BaS, cioè due espressioni presenti nella tavola di verità, prima della minimizzazione. Anche BA implica l'espressione BAS ma non BaS, mentre implica BAs, questo vuol dire che per coprire tutte le espressioni derivate dalla tavola di verità sono necessari diversi primi implicanti. 
Nel caso qui discusso tutti i primi implicanti sono necessari ma in alcuni casi può succedere che essi siano ridondanti, ossia in eccesso, rispetto alla necessità di coprire tutte le espressioni, per cui può essere utile trovare qual è il loro numero minimo necessario. Per fare ciò si utilizza un altro strumento di minimizzazione, una griglia dei primi implicanti di cui la fig.3 è un esempio:

\begin{tabular}{llllll}
\hline Primi implicanti & Espressioni originarie & Espressioni originarie & Espressioni originarie & Espressioni originarie & Espressi \\
\hline & BAS & BaS & BAs & Bas & bas \\
BS & $\mathrm{x}$ & $\mathrm{x}$ & $\mathrm{x}$ & $\mathrm{x}$ & $\mathrm{x}$ \\
BA & & & & & \\
as & & & & &
\end{tabular}

Fig.3

Esaminando la griglia, appare evidente che per coprire le cinque espressioni originate dalla tavola di verità occorrono tutti e tre i primi implicanti ma se, per esempio, escludessimo l'espressione BAs, allora BS e as sarebbero sufficienti da soli a coprire le rimanenti quattro espressioni originarie.

\section{Conclusioni}

Proviamo adesso ad esporre in termini meno astratti cosa vuol dire l'espressione algebrica ottenuta, $\mathrm{PF}=$ $\mathrm{BS}+\mathrm{BA}+$ as.

L'equazione afferma che la fragilità dei pazienti elencati dai MMG può essere causata dalla polipatologia e/o politerapia assieme (AND) alla solitudine ( assenza di conviventi e rete sociale attiva) senza che siano necessarie altre cause, oppure (OR) dalla polipatologia e/o politerapia anche (AND) in un paziente pienamente autonomo,oppure (OR) in un paziente non autonomo anche (AND) se convivente con qualcuno.

Naturalmente ci possono essere altre combinazioni in altri casi empirici di pazienti considerati fragili ma l'uso dell'Analisi Quantitativa Comparata enfatizza l'aspetto congiunturale e multiplo delle cause in quanto esse sono combinazioni di fattori e molte di queste combinazioni possono dare lo stesso risultato.

Da una ricerca convenzionale sul paziente fragile ci si può aspettare un'equazione che spieghi quanto la fragilità sia dovuta, rispettivamente, alla polipatologia, alla politerapia, alla disabilità, alla solitudine o alla povertà. Con un classico metodo quantitativo quando si esaminano casi diversi di pazienti fragili i valori delle variabili possono cambiare ma la struttura dell'equazione deve rimanere la stessa, pena la sua validità.

Nell'analisi booleana ci si aspetta invece di trovare più di un insieme di condizioni che diano come risultato la fragilità di un paziente. Fattori diversi possono combinarsi fra loro in modi diversi, perfino contradditori, dando risultati simili e poiché può accadere che non tutte le condizioni necessarie ad una spiegazione siano considerate, allora la spiegazione può non tener conto di tutti i casi.11Becker H.S., (op.cit.)

Nello studio qui riportato, i fattori causali sono quelli indicati dai MMG per i casi empirici di pazienti fragili da essi individuati e l'espressione ottenuta si riferisce ad essi, ciò non toglie che altri MMG potrebbero includere altre cause per la fragilità che però non andrebbero ad inficiare la validità del metodo ma semplicemente allargherebbero la "rosa" delle possibili combinazioni. Il metodo utilizzato nell'AQC non fornisce una spiegazione della fragilità ma aiuta a comprendere, nel senso letterale di prendere assieme, le varie coincidenze congiunturali delle cause.

Oltre a sottolineare questo aspetto congiunturale, l'AQC mediante l'utilizzo della tavola di verità, garantisce all'analista che non venga trascurata nessuna delle combinazioni di cause logicamente possibili.

Il carattere olistico dell'AQC consente di guardare alle cause che determinano il paziente fragile in termini combinatori, come un tutto, e non come semplice sommatoria di fattori diversi ed anche il confronto fra le varie cause avviene in modo olistico. 
Inoltre, basandosi sull'algebra booleana, l'approccio dell'AQC procede induttivamente, secondo una traiettoria bottom-up, semplificando la complessità dei casi di PF considerati, in modo metodico e graduale.

Infine, la possibilità di esprimere le condizioni causali in termini di necessità e sufficienza facilita l'analisi comparativa soprattutto in quelle situazioni in cui cause diverse possono dare risultati simili o uguali.

In conclusione, si può affermare che la AQC può risultare utile anche in ambiti come quello medico nel quale sono frequenti le situazioni complesse che vanno affrontate in modo olistico per poter ridurne la complessità a favore di un'analisi più efficace.

Saranno necessarie ulteriori verifiche della sua applicazione in questo campo, magari in situazioni cliniche e socio-sanitarie maggiormente definite e consolidate rispetto a quella del paziente fragile, che comunque resta un ambito nel quale l' AQC dimostra di poter fornire uno strumento valido di gestione della complessità.

Ringraziamenti: desidero ringraziare i medici di Medicina Generale Mariateresa Gallea, Francesca Mungo, Francesco Cavasin, Umberto De Conto e Stefano Ivis per la collaborazione offerta.

1. Zucchelli G., Silvi G., La fragilità dell'anziano , Livorno, Debatte editore, 2001

2. Gobbens RJJ, Luijkx KG, Wijnen-Sponselee M, et al.Insearch of an integral conceptual definition of frailty:opinions of experts. J Am Med Dir Assoc 2010;11:338-43.

3. 